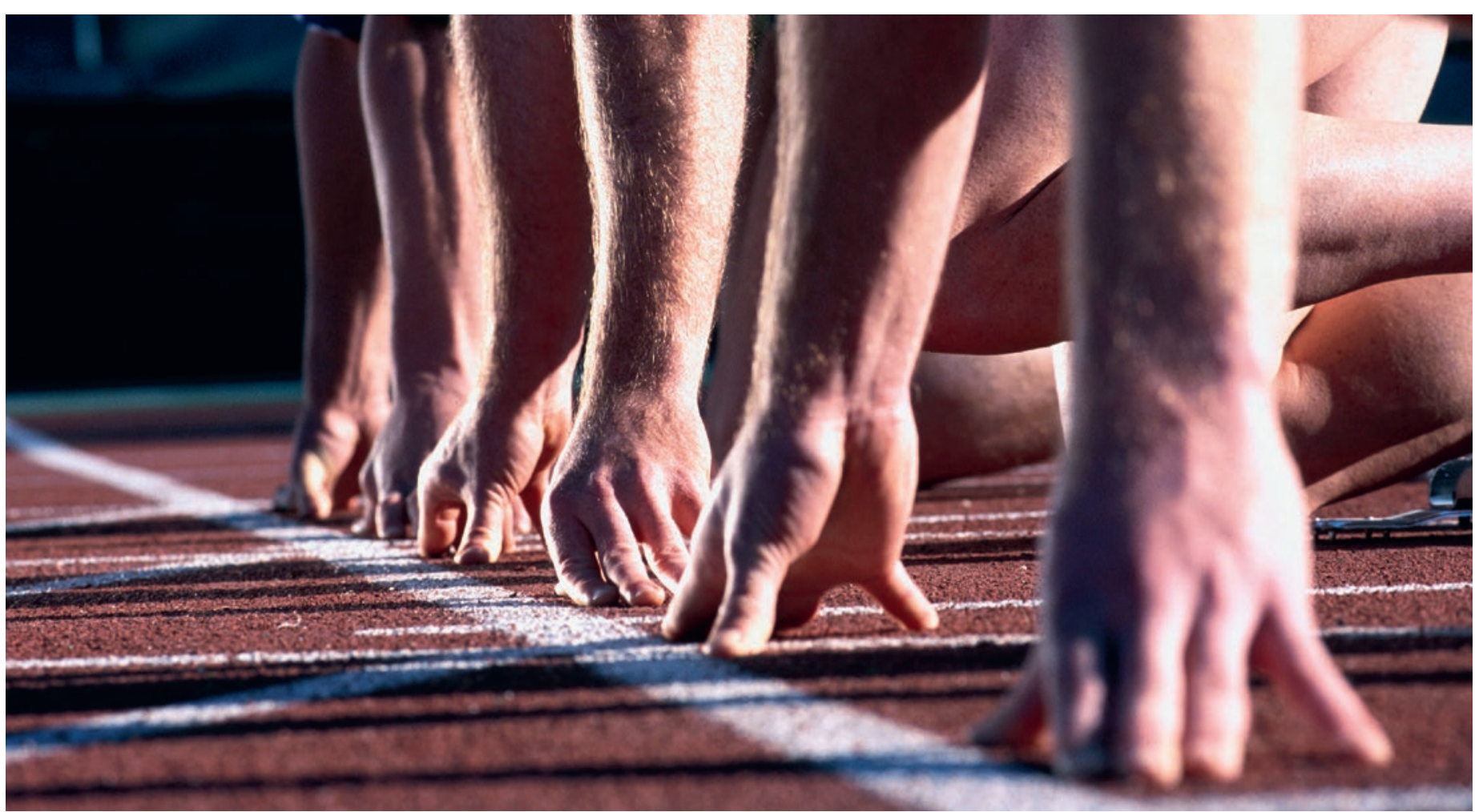

\title{
Antidoping Suisse devient Swiss Sport Integrity
}

\section{Carina Brunner}

Pharmacienne dipl. féd., Fondation Swiss Sport Integrity, Berne

Depuis le début de l'année, le domaine de compétence et le but de la Fondation Antidoping Suisse ont été élargis: l'agence nationale antidopage s'appelle désormais Swiss Sport Integrity. En outre, depuis le $1^{\text {er }}$ janvier 2022, des règles plus strictes s'appliquent aux glucocorticoïdes.

L'excellence, l'amitié et le respect: les valeurs olympiques caractérisent le sport au niveau international et jouent également un rôle prépondérant dans la vie quotidienne. Cependant, depuis plusieurs années, les méthodes d'entraînement dépourvues d'éthique dans le sport suisse font de plus en plus l'objet de reproches. La responsabilité appartenait jusqu'à présent aux fédérations sportives qui étaient tenues d'identifier les comportements déviants et les abus au sein de leur propre organisation et, le cas échéant, de prononcer des sanctions. Cette responsabilité a désormais été transférée: avec Swiss Sport Integrity, le sport suisse dispose d'un centre d'aide indépendant et fiable, chargé de détecter et d'éliminer le plus rapidement possible les manquements à l'éthique et les abus. Swiss Sport Integrity poursuit les tâches et les activités d'Antidoping Suisse et continue à assumer son rôle d'organisation nationale antidopage.

\section{Portail de signalement}

Le portail de signalement de Swiss Sport Integrity est à la disposition de toutes les personnes qui souhaitent signaler d'éventuelles violations ou abus via www. 
sportintegrity.ch. Une consultation de premier recours permet d'informer sur les possibilités de procédure et, le cas échéant, de diriger les personnes vers un centre de conseil compétent pour une consultation approfondie. Le signalement reçu sera examiné et, si nécessaire, transmis aux autorités de poursuite pénale. Si la compétence revient à Swiss Sport Integrity, une enquête sera réalisée et un rapport final sera rédigé à l'attention de la Chambre disciplinaire du sport suisse qui se prononcera sur d'éventuelles sanctions.

\section{Glucocorticoïdes: règles plus strictes}

Pour la Liste des interdictions 2022, l'Agence mondiale antidopage (AMA) a effectué des adaptations qui ont des conséquences sur le statut d'interdiction des médicaments. Celles-ci concernent en particulier les glucocorticoïdes. En sus de l'administration par voie orale, intraveineuse, intramusculaire ou rectale, toute sorte d'injection est désormais interdite en compétition. Si une substance est interdite "en compétition", son usage pendant cette période ou sa détection lors d'un contrôle antidopage en compétition, entre autres, est considéré comme une violation des règles antidopage. Cela ne s'applique pas si une autorisation d'usage à des fins thérapeutiques (AUT) a été accordée ou peut être accordée rétroactivement.

Les injections locales peuvent causer des concentrations similaires à celles obtenues par l'administration systémique de glucocorticoïdes.

L'interdiction a été étendue étant donné que les injections locales de glucocorticoïdes peuvent provoquer des concentrations plasmatiques et urinaires similaires à celles obtenues par l'administration systémique de glucocorticoïdes. On peut donc supposer que les injections locales présentent à la fois une amélioration potentielle de la performance sportive et des risques pour la santé comparables [1]. D’autres voies d'administration (par ex. inhalation, application topique) des glucocorticoïdes restent permises en et hors compétition.

A l'aide de la base de données sur les médicaments DRO global (via www.sportintegrity.ch/fr/medicaments ou Medi-Check App), le statut d'interdiction des médicaments peut être vérifié en tenant compte de la voie d'administration.

\section{Tenir compte des périodes de sevrage}

Le délai entre l'usage d'un glucocorticoïde et son excrétion dépend de la substance, de la voie d'administration et du dosage. Ce qui signifie qu'après l'utilisation d'un glucocorticoïde, la période pendant laquelle, lors d'un contrôle antidopage en compétition, un échantillon s'avère positif peut varier notablement. L'AMA a donc défini les périodes de sevrage pour les glucocorticoïdes interdits en compétition [2]. Les périodes de sevrage respectives applicables par substance et voie d'administration (dans une fourchette de 3 à 60 jours) sont publiées sur www.sportintegrity.ch/fr/glucocorticoides et, pour les substances et les médicaments concernés, les périodes de sevrage sont affichées dans la base de données sur les médicaments DRO global.

\section{Directives d'action}

Si, lors de l'usage thérapeutique précédant une compétition, les périodes de sevrage sont respectées, un résultat d'analyse positif en compétition est hautement improbable.

Si la période de sevrage avant une compétition ne peut pas être respectée ou si le glucocorticoïde est utilisé en compétition, il faut respecter les directives d'action respectives sur www.sportintegrity.ch/fr/glucocorticoides. Dans toutes les situations, il est très important pour les athlètes que le diagnostic et le choix de la thérapie soient justifiés et documentés de manière détaillée par le médecin traitant.

\section{Crédits photo}

Explorer Media Pty Ltd Sport The Library | Dreamstime.com

\section{Références}

1 Ventura R, Daley-Yates P, Mazzoni I, Collomp K, Saugy M, Buttgereit F, et al. A novel approach to improve detection of glucocorticoid doping in sport with new guidance for physicians prescribing for athletes. Br J Sports Med. 2021 Apr 20. doi: 10.1136/bjsports-2020-103512

2 www.wada-ama.org/sites/default/files/resources/files/2022list_ explanatory_note_final_en.pdf

\section{L'essentiel en bref}

- Depuis janvier 2022, la Fondation Antidoping Suisse s'appelle Swiss Sport Integrity. Elle se charge désormais (en plus des tâches antidopage) d'enquêter sur les signalements de manquements à l'éthique.

- Pour ce faire, elle a mis en place un portail de signalement qui est ouvert à toutes les personnes souhaitant signaler d'éventuelles violations ou irrégularités.

- L'Agence mondiale antidopage (AMA) a effectué des adaptations qui ont des conséquences sur le statut d'interdiction des médicaments. Pour les glucocorticoïdes, toute injection est désormais interdite en compétition.

- Des informations sur les périodes de sevrage pour les glucocorticoïdes interdits en compétition se trouvent sur www.sportintegrity.ch/fr/glukokortikoide et sont publiées dans la base de données sur les médicaments DRO global. 